Tailored Anodization Leads to the Formation of Long, High-AspectRatio $\mathrm{TiO}_{2}$ Nanotube Arrays for Dye-Sensitized Solar Cells

As dye-sensitized solar cells (DSCs) continue to receive significant attention for their potential as low-cost alternatives to crystalline silicon photovoltaics, it appears that arrays of nanotubular $\mathrm{TiO}_{2}$ may hold the key to their performance. In particular, the use of long, smooth $\mathrm{TiO}_{2}$ nanotube arrays instead of disordered nanoparticulate $\mathrm{TiO}_{2}$ can enhance DSC efficiencies by absorbing larger amounts of photosensitizing dye, leading to increased photoelectron generation. Now, work reported in the September issue of the Journal of the American Ceramic Society (DOI: 10.1111/j.1551-2916.2008.02546.x; p. 3086) by Y. Yang, X. Wang, and L. Li at Tsinghua University in Beijing demonstrates that careful control of the conditions of anodization can lead to the formation of smooth $\mathrm{TiO}_{2}$ nanotube arrays as long as $22 \mu \mathrm{m}$, with aspect ratios up to 200 .

The researchers began the nanotube formation process by potentiostatically anodizing $99.5 \%$-pure titanium foils in a dimethylformamide electrolyte solution containing $0.5 \mathrm{wt} \%$ hydrofluoric acid, under an applied voltage of $30 \mathrm{~V}$. This resulted in arrays of $\mathrm{TiO}_{2}$ nanotubes whose length increased with anodization time, saturating at $22 \mu \mathrm{m}$ after $40 \mathrm{~h}$, which the researchers identify as the point at which the oxide growth and dissolution rates equalize. The nanotubes' wall smoothness also increased with anodization time, and their aspect ratio was as high as 200, as revealed in fieldemission scanning electron microscope images. The researchers next created samples using $50 \mathrm{~h}$ of anodization time and different (fixed) applied voltages, finding that the average nanotube diameter increased from $110 \mathrm{~nm}$ at $20 \mathrm{~V}$ to $130 \mathrm{~nm}$ at $30 \mathrm{~V}$. A key observation of these experiments was that the nanotubes were covered by a thick protective oxide layer during the anodization, enabling the long growth.

In order to investigate the effectiveness of these long nanotube arrays in solar cells, the researchers first calcined them at $400^{\circ} \mathrm{C}$ for $3 \mathrm{~h}$ and then used them as photoanodes in DSC devices. Although the devices employed an inexpensive and less-effective dye (Indoline dye D102) than the state of the art, the device's conversion efficiency was as high as $3.4 \%$. In light of these results, long, high-aspectratio $\mathrm{TiO}_{2}$ nanotube arrays may bring the commercial viability of DSCs one step closer to reality.

COLIN MCCORMICK

\section{Morphology and Optical Response of Butterfly Wing Replicated as an Inorganic Glass}

The objective of biomimetics is to take advantage of structures found in nature, which have been optimized over millions of years of evolution, for the design of new materials and devices. Biomimetics researchers have been attracted to structures found in insects because they display characteristics that simultaneously serve several functions. For example, the structure of butterfly wings, in addition to making flight possible, is generally responsible for the great diversity of colors and patterns that exist. In one approach to fabricating replicas of biological structures, actual biological samples serve as templates that are coated with inorganic materials such that the microstructure and nanostructure are captured. Butterfly wings are covered with scales to a density of 200-600 scales/ $\mathrm{mm}^{2}$ arranged in a roof-tile pattern. The replicated scale patterns are complex in structure with stratification, voids, and complex groove shapes that can result in a photonic bandgap structure. A biomimetic

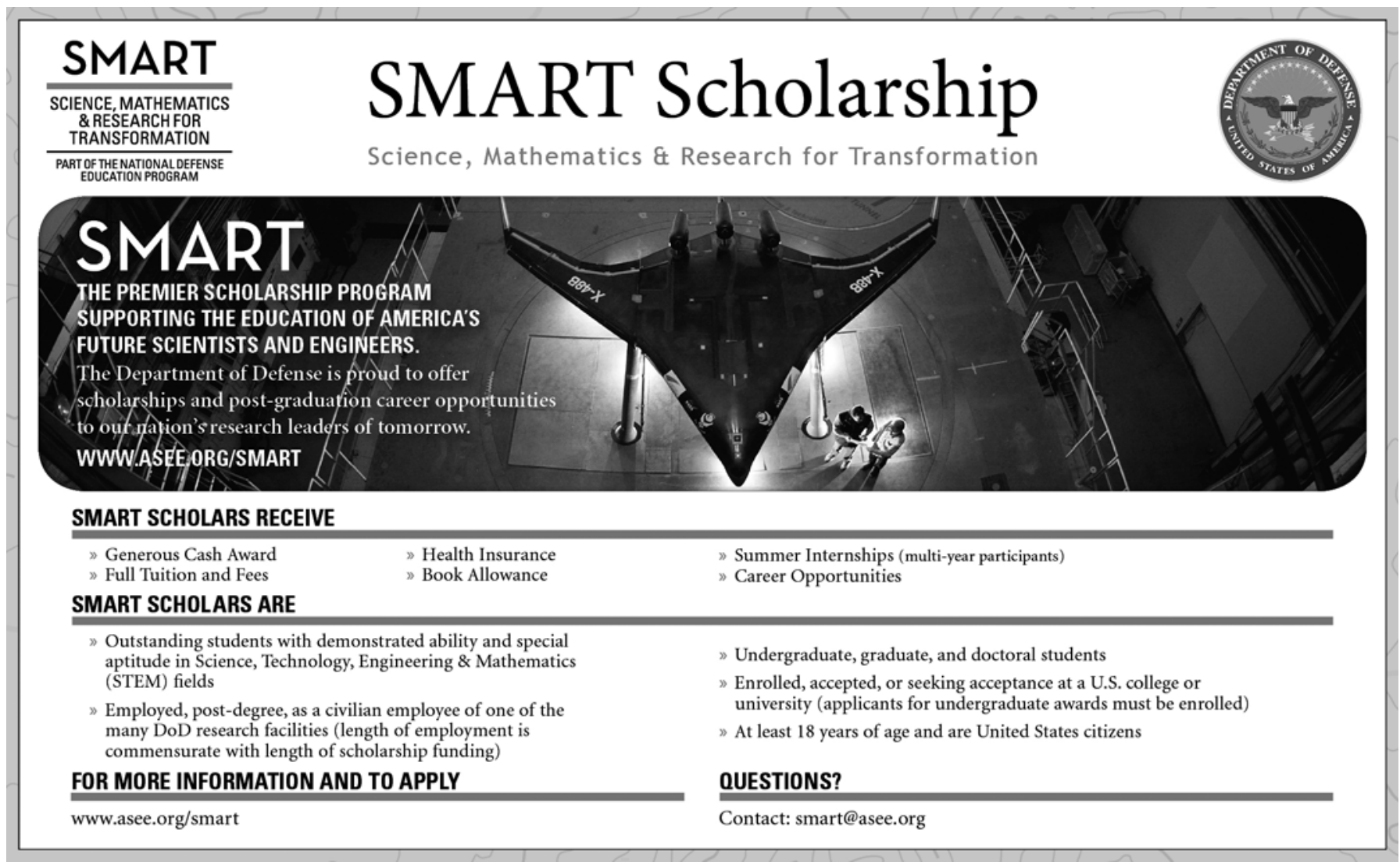


technique, called conformal-evaporatedfilm-by-rotation (CEFR), recently developed by R.J. Martín-Palma, C.G. Pantano, and A. Lakhtakia of The Pennsylvania State University, involves thermally evaporating an inorganic glass onto a rotating biological sample, to replicate at the microand nanoscale the morphology of a butterfly wing.

As reported recently in Applied Physics Letters (DOI: 10.1063/1.2973167), the researchers replicated a wing of the butterfly Battus philenor with chalcogenide glass $\left(\mathrm{Ge}_{28} \mathrm{Sb}_{12} \mathrm{Se}_{60}\right)$, chosen because of its large index of refraction at visible and infrared (IR) wavelengths, and its high transmittance in the IR. In addition, illumination of chalcogenide glasses can modify the optical bandgap and index of refraction, making them suitable for passive and active infrared devices. The researchers thermally evaporated the chalcogenide glass using a current of $95 \mathrm{~A}$ for $30 \mathrm{~min}$, directing the vapor flux toward the substrate at an angle of $85^{\circ}$ to normal, with the substrate rotating at a constant speed of 0.5 rotations/s, resulting in a coating about $0.5 \mu \mathrm{m}$ thick. Scanning electron microscope images show that the replicated structure is composed of thousands of scales with typical dimensions of about $50 \mu \mathrm{m}$ by $200 \mu \mathrm{m}$, arranged in rows. A grid of raised longitudinal, quasiparallel lamellae runs the length of a typical scale, spaced at about $2.5 \mu \mathrm{m}$. Filling the space between adjacent lamellae is a netlike reticulum, composed of fine tubes. The researchers said that both the lamellae and the reticulum are the optical elements that give the wing its particular color. The researchers used energy dispersive $\mathrm{x}$-ray spectroscopy to show that they fabricated a high-fidelity, chalcogenide glass replica of the original biotemplate without disturbing the original structure. In addition, specular and scattered reflectance spectra in the visible and IR (200 nm-2 $\mu \mathrm{m})$ are similar for the original specimen and the replica; the minor differences were explained by the researchers as due to the difference in the indices of refraction of the wing material and the glass. In addition, the researchers said, "Since the morphology of the butterfly wing makes it a very efficient diffuser of light, the replica could be used as an antireflection structure for increased photon trapping and optical diffusers," and "the CEFR technique might be useful for the development of high-efficiency biomimetic optical devices."

STEVEn TrohalaKi

\section{Higher Order Stop Gaps Observed in Photosensitive Chalcogenide Glasses 3D Photonic Crystals}

Photonic crystals (PCs) are key components for the next generation of miniatur- ized photonic devices because of their capability to control and manipulate the flow of light on a wavelength scale. E. Nicoletti and co-workers from the Swinburne University of Technology, Victoria, and D. Bulla and co-workers from the Australian National University, Canberra, have reported in a recent issue of Optics Letters (posted online on September 8) on the observation of first- and second-order stop gaps in the near-infrared wavelength region on three-dimensional (3D) PCs based on photosensitive chalcogenide glasses (ChGs) fabricated by directlaser-writing.

The researchers used $~ 16-20-\mu m$-thick $\mathrm{As}_{2} \mathrm{~S}_{3}$ films deposited on glass slide substrates by thermal evaporation of $\mathrm{As}_{2} \mathrm{~S}_{3}$ bulk glass in a vacuum chamber at $2 \times 10^{-7}$ Torr and $310^{\circ} \mathrm{C}$. Such films contain disconnected molecular cagelike structures (e.g., $\mathrm{As}_{4} \mathrm{~S}_{4}$, $\mathrm{As}_{4} \mathrm{~S}_{6}$ ) that can "open" when thermally or optically excited. The researchers produced this effect using a femtosecond Ti:sapphire laser beam (wavelength $800 \mathrm{~nm}$, repetition rate $1 \mathrm{kHz}$ ), tightly focused into the films, leading to re-bonding and polymerization of the glass network in highly localized areas of the glass through a two-photoninduced nonlinear process. Subsequently, the researchers dissolved the unpolymerized glass in a solution of diisopentylamine and dimethylsulfoxide in multiple steps, starting with a highly concentrated

\section{Advertisers in This Issue}

\begin{tabular}{|c|c|c|c|}
\hline \multicolumn{4}{|c|}{ Advertisers in This Issue } \\
\hline & Page No. & & Page No. \\
\hline *A \& N Corporation & 1066 & *Janis Research Company, Inc. & 991 \\
\hline *Agilent Technologies & 1001 & *JEOL USA Inc. & 1026 \\
\hline *Aldrich/Sigma-Aldrich & $\overline{1046}$ & *Kurt J. Lesker Company & 1035 \\
\hline ^Angstrom Sciences & 993 & *Lake Shore Cryotronics, Inc. & $\overline{1068}$ \\
\hline ^Annealsys & 992 & ^Leica Microsystems Inc. & $\overline{1060}$ \\
\hline *Asylum Research & 986 & *MDC Vacuum Products, LLC & 1052 \\
\hline *Bruker AXS Inc. & $\overline{1016}$ & *MMR Technologies, Inc. & 992 \\
\hline *Carl Zeiss SMT Inc. & 1036 & NanoScale Science at UNC Charlotte & 1074 \\
\hline *CrystaIMaker Software Ltd. & 1058 & National Electrostatics Corp. & 1000 \\
\hline *Digital Surf SARL & 991 & *NT-MDT Co. & 1069 \\
\hline *Gatan, Inc. & 1015 & *Park Systems, Inc. & Inside back cover \\
\hline *Goodfellow Corporation & 1051 & Radcliffe Fellowships & 997 \\
\hline High Voltage Engineering Europa B.V. & Inside front cover & ${ }^{*}$ Royal Society of Chemistry & $\overline{1067}$ \\
\hline *Huntington Mechanical Laboratories, Inc. & Outside back cover & SMART Scholarship/ASEE & 989 \\
\hline *Hysitron, Inc. & 994 & *Veeco Instruments Inc. & 985 \\
\hline *International Centre for Diffraction Data (ICDD) & 1002 & Visitec of America & 1059 \\
\hline
\end{tabular}

For the direct link to advertisers in this issue, access www.mrs.org/bulletin_ads.

*Please visit us at the Exhibit, December 2-4, during the 2008 MRS Fall Meeting in Boston. 